\title{
Refractive Indices of Liquid Crystals for Display Applications
}

\author{
Jun Li, Student Member, IEEE, Chien-Hui Wen, Sebastian Gauza, Ruibo Lu, and Shin-Tson Wu, Fellow, IEEE
}

\author{
Invited Paper
}

\begin{abstract}
This paper reviews the extended Cauchy model and the four-parameter model for describing the wavelength and temperature effects of liquid crystal (LC) refractive indices. The refractive indices of nine commercial LCs, MLC-9200-000, MLC-9200-100, MLC-6608, MLC-6241-000, 5PCH, 5CB, TL-216, E7, and E44 are measured by the Multi-wavelength Abbe Refractometer. These experimental data are used to validate the theoretical models. Excellent agreement between experiment and theory is obtained.
\end{abstract}

Index Terms-Cauchy model, four-parameter model, liquid crystal display (LCD), liquid crystal (LC) refractive indices, temperature gradients, three-parameter parabolic model.

\section{INTRODUCTION}

$\mathbf{T}$ HIN-FILM transistor liquid crystal display (TFT-LCD) has been commonly used in direct-view, e.g., cellular phones, notebook and desktop computers, and televisions, and large screen projection displays [1], [2]. The fundamental light modulation mechanism of a LCD is electric field-induced molecular reorientation which, in turn, causes refractive index change. The refractive indices of a liquid crystal (LC) are mainly determined by the molecular structure, wavelength, and operating temperature. To achieve a full-color display three primary colors (red, green, and blue) are needed. As the wavelength increases, the refractive indices decrease. The decreasing rate depends on the LC molecular structures. On the other hand, for a front or rear projection display, the thermal effect originated from the lamp heating raises the LC panel temperature to $50^{\circ} \mathrm{C}-60^{\circ} \mathrm{C}$. To design the $\mathrm{LC}$ panels for projection displays, we need to know the intended operating temperature. As the temperature increases, the extraordinary refractive index decreases monotonously, but the ordinary refractive index could decrease or increase depending the crossover temperature of the LC employed. Therefore, the wavelength- and temperature-dependent refractive indices are fundamentally interesting and practically important for optimizing the display performances and other photonic devices employing LCs.

Manuscript receivedFebruary 5, 2005; revised June 16, 2005. This work was supported by the Air Force Office of Scientific Research under Contract F49620-01-1-0377 and by Toppoly Optoelectronics (Taiwan).

The authors are with the College of Optics and Photonics, University of Central Florida, Orlando, FL 32816 USA (e-mail: junli@mail.ucf.edu; vicky@ mail.ucf.edu; sgauza@mail.ucf.edu; rlu@mail.ucf.edu; swu@mail.ucf.edu).

Digital Object Identifier 10.1109/JDT.2005.853357
Several physical models have been developed to describe the wavelength and temperature dependencies of LC refractive indices [3]-[8]. Each model has its own merits and incompleteness. For instances, the Vuks model [3] correlates the microscopic molecular polarizability to the macroscopic refractive indices, but the wavelength and temperature effects are not described explicitly. The single band model [5], [6] gives an explicit expression on the wavelength and temperature dependence for birefringence, but not for the individual refractive indices. The three-band model [7] describes the origins of the refractive indices for single LC compounds but requires three fitting parameters for each LC compound. If an LC mixture consists of a dozen different molecular structures, it would be too complicated for the three-band model to describe the LC refractive indices of the mixture quantitatively.

Although the original Cauchy equation [9] was intended for the isotropic gases and liquids, it has been attempted to fit the wavelength-dependent refractive indices of some anisotropic LCs [10], [11]. The fitting results are reasonably good except that the physical origins of the Cauchy coefficients are not clear. Recently, the extended Cauchy equations [12], [13] were derived based on the three-band model for fitting the refractive indices of anisotropic LCs [6], [7]. The extended Cauchy equations are applicable not only to single compounds but also to LC mixtures. Good fittings are found in the off-resonance region. If the LC birefringence is low, then the three coefficients in the extended Cauchy model can be reduced to two and the fitting process is simpler. To describe the temperature effect of LC refractive indices, a four-parameter model has been derived based on Vuks equation [3]. If the LC clearing temperature is high, the four-parameter model can be further simplified to the three-parameter parabolic model [14].

In this review article, we briefly present the extended Cauchy model and the two-coefficient Cauchy model for describing the wavelength effect of the LC refractive indices. We also discuss the four-parameter and three-parameter parabolic models for describing the temperature effect on the refractive indices of LCs. In Section II, we review the derivation processes for these models. In Section III, the UV absorption spectra and refractive indices of four commercial TFT LC mixtures: MLC-9200-000, MLC-9200-100, MLC-6608, and TL-216, were measured. To find the boundary value of birefringence below which the twocoefficient Cauchy model works equally well as the three-coefficient Cauchy model, the refractive indices of five more LCs: 
E44, E7, 5CB, 5PCH, and MLC-6241-000 were measured. In Section IV, we validate the physical models by fitting the experimental data of these LC mixtures. Excellent agreement between theory and experiment is obtained. Finally, the temperature nonlinearity of LCs is discussed.

\section{THEORY}

The aligned LC exhibits anisotropic properties, such as optical, dielectric, and elastic anisotropies. In a homogeneous cell, the LC exhibits two refractive indices $n_{e}$ and $n_{o}$, depending on the incident light polarization. If the incident light is linearly polarized light parallel to the LC directors, i.e., extraordinary ray, the corresponding refractive index is $n_{e}$. On the other hand, if the incident linearly polarized light is perpendicular to the LC directors, i.e., ordinary ray, the corresponding refractive index is $n_{o}$. Birefringence is defined as $\Delta n=n_{e}-n_{o}$. Except for some special molecular structures [15], most LC compounds and mixtures possess a positive birefringence, i.e., $n_{e}>n_{o}$.

For active matrix display applications, the LC cell gap $(d)$ is usually in the 3-5 $\mu \mathrm{m}$ range. The detailed selection depends on the balance between response time and manufacturing yield. A narrower cell gap leads to a faster response time, but the manufacturing yield could be sacrificed. In addition, a smaller cell gap would require a higher birefringence to satisfy the minimum $d \Delta n$ requirement. A high birefringence $\mathrm{LC}$ tends to have less photo and thermal stability. For most transmission-type displays developed so far, the required LC birefringence is around $0.10-0.15$. For reflective displays, the incident light traverses the LC layer twice [16] so that the $d \Delta n$ value can be reduced by nearly half. Other desirable parameters include low viscosity, modest dielectric anisotropy, proper elastic constants, and good material stability for long-term operation.

Wavelength, temperature, and molecular constituents play key roles in determining the LC refractive indices. Here, we discuss the wavelength and temperature effects.

\section{A. Wavelength Effect}

The major absorption of a LC compound occurs in two spectral regions: ultraviolet (UV) and infrared (IR). [17], [18] The $\sigma \rightarrow \sigma^{*}$ electronic transitions take place in the vacuum UV $(100-180 \mathrm{~nm})$ region whereas the $\pi \rightarrow \pi^{*}$ electronic transitions occur in the UV (180-400 nm) region. If a LC compound has a longer conjugation, its electronic transition wavelength would extend to a longer UV wavelength. In the near IR region, some overtone molecular vibration bands appear. [17], [18] The fundamental molecular vibration bands, such as $\mathrm{CH}, \mathrm{CN}$, and $\mathrm{C}=\mathrm{C}$, occur in the mid and long IR regions. Typically, the oscillator strength of these vibration bands is about two orders of magnitude weaker than that of the electronic transitions. Thus, the resonant enhancement of these bands to the LC birefringence is localized [17], [18].

The three-band model [6], [7] was derived based on the LC absorption spectra by taking the three main electronic transitions into consideration: one $\sigma \rightarrow \sigma^{*}$ transition (the $\lambda_{0}$-band) and two $\pi \rightarrow \pi^{*}$ transitions (the $\lambda_{1}$ - and $\lambda_{2}$-bands). In the three-band model, the refractive indices $\left(n_{e}\right.$ and $\left.n_{o}\right)$ are expressed as follows [6], [7]:

$$
\begin{aligned}
& n_{e} \cong 1+g_{0 e} \frac{\lambda^{2} \lambda_{0}^{2}}{\lambda^{2}-\lambda_{0}^{2}}+g_{1 e} \frac{\lambda^{2} \lambda_{1}^{2}}{\lambda^{2}-\lambda_{1}^{2}}+g_{2 e} \frac{\lambda^{2} \lambda_{2}^{2}}{\lambda^{2}-\lambda_{2}^{2}} \\
& n_{o} \cong 1+g_{0 \circ} \frac{\lambda^{2} \lambda_{0}^{2}}{\lambda^{2}-\lambda_{0}^{2}}+g_{1 o} \frac{\lambda^{2} \lambda_{1}^{2}}{\lambda^{2}-\lambda_{1}^{2}}+g_{2 o} \frac{\lambda^{2} \lambda_{2}^{2}}{\lambda^{2}-\lambda_{2}^{2}} .
\end{aligned}
$$

The three-band model describes the origins of refractive indices of LC compounds. However, a commercial mixture usually consists of several compounds with different molecular structures in order to obtain wide nematic range. The individual $\lambda_{i}$ 's are, therefore, different so that (1) would have too many unknowns to describe the refractive indices of a LC mixture.

To model the refractive indices of a LC mixture, we could expand (1) into power series because in the visible and IR spectral regions, $\lambda \gg \lambda_{2}$. By keeping up to the $\lambda^{-4}$ terms, we derive the extended Cauchy model [12], [13]:

$$
\begin{aligned}
& n_{e} \cong A_{e}+\frac{B_{e}}{\lambda^{2}}+\frac{C_{e}}{\lambda^{4}} \\
& n_{o} \cong A_{o}+\frac{B_{o}}{\lambda^{2}}+\frac{C_{o}}{\lambda^{4}} .
\end{aligned}
$$

Although (2) is derived based on a LC compound, it can be extended easily to include eutectic mixtures by taking the superposition of each compound [13]. From (2), if we measure the refractive indices at three wavelengths, the three Cauchy coefficients $\left(A_{e, o}, B_{e, o}\right.$ and $\left.C_{e, o}\right)$ can be obtained by fitting the experimental results. Once these coefficients are determined, the refractive indices at any wavelength can be calculated. From (2), the refractive indices and birefringence decrease as the wavelength increases. In the long wavelength (IR and millimeter wave) region, $n_{e}$ and $n_{o}$ are reduced to $A_{e}$ and $A_{o}$, respectively. The coefficients $A_{e}$ and $A_{o}$ are constants; they are independent of wavelength, but dependent on the temperature. That means, in the IR region the refractive indices are insensitive to wavelength, except for the resonance enhancement effect near the local molecular vibration bands [5].

Equation (2) applies equally well to both high and low birefringence LC materials in the off-resonance region. For low birefringence LC mixtures, the $\lambda^{-4}$ terms are insignificant and can be omitted. Thus, $n_{e}$ and $n_{o}$ each has only two parameters [18]. The two-coefficient Cauchy model has the following simple forms [13]:

$$
n_{e, o} \cong A_{e, o}+\frac{B_{e, o}}{\lambda^{2}}
$$

By measuring the refractive indices at two wavelengths, we can determine $A_{e, o}$ and $B_{e, o}$. Once these parameters are found, we can calculate the $n_{e}$ and $n_{o}$ at any wavelength of interest.

\section{B. Temperature Effect}

The temperature effect is particularly important for projection displays. Due to the thermal effect of the lamp, the temperature of the display panel could reach $50^{\circ} \mathrm{C}-60^{\circ} \mathrm{C}$. It is important to know the LC properties at the anticipated operating temperature beforehand. The thermal nonlinearity of LC refractive indices is also very important for some new photonic applications, such as LC photonic bandgap fibers [19], [20] and thermal soliton [21], [22]. 
Several physical models have been developed to describe the temperature effect of the LC refractive indices. The classical Clausius-Mossotti equation correlates the permittivity $(\varepsilon)$ of an isotropic media with molecular polarizability $(\alpha)$ as follows [23]:

$$
\frac{\varepsilon-1}{\varepsilon+2}=\frac{4 \pi}{3} N \alpha .
$$

In (4), $N$ is the molecular packing density, or number of molecules per unit volume. In the optical frequency regime, we substitute $\varepsilon=n^{2}$ and obtain the Lorentz-Lorenz equation [23]:

$$
\frac{n^{2}-1}{n^{2}+2}=\frac{4 \pi}{3} N \alpha
$$

For an anisotropic LC, there are two principal refractive indices, $n_{e}$ and $n_{o}$. In principle, each refractive index should be related to its corresponding molecular polarizability, $\alpha_{e}$ and $\alpha_{o}$. An early approach replaces both $n^{2}$ in (5) by $n_{e, o}^{2}$ and $\alpha$ by $\alpha_{e, o}$ [3]. However, this approach does not fit the experimental results well. Vuks made a bold assumption that the internal field in a crystal is the same in all directions. With this assumption, Vuks derived the following equation for anisotropic media [3]:

$$
\frac{n_{e, o}^{2}-1}{\left\langle n^{2}\right\rangle+2}=\frac{4 \pi}{3} N \alpha_{e, o} .
$$

Equation (6) is different from (5) in two aspects: 1) the $n^{2}$ term in the denominator of (5) is replaced by $\left\langle n^{2}\right\rangle=\left(n_{e}^{2}+2 n_{o}^{2}\right) / 3$ while the $n^{2}$ term in the numerator is replaced by $n_{e, o}^{2}$ and 2) the $\alpha$ is replaced by $\alpha_{e, o}$. Vuks validated (6) by using several anisotropic crystals such as naphthalene, diphenyl, anthracene, and phenanthrene [3]. From our earlier work, we found that $\left\langle n^{2}\right\rangle$ is linearly proportional to $\langle n\rangle$ as [24]

$$
\left\langle n^{2}\right\rangle+2 \approx \sqrt{10}\langle n\rangle-0.5
$$

It is easy to validate (7) since both $\langle n\rangle$ and $\left\langle n^{2}\right\rangle$ are macroscopic observables. Equation (7) has been validated by the experimental data of several LC materials [24]. Thus, the Vuks isotropic local field assumption is self-consistent.

Based on the Vuks equation, the following expressions for $n_{e}$ and $n_{o}$ can be derived [24]

$$
\begin{aligned}
& n_{e} \approx \frac{7}{2 \sqrt{10}}+\frac{\frac{2 \sqrt{10}}{5} \pi N\langle\alpha\rangle}{1-\frac{4}{3} \pi N\langle\alpha\rangle}+\frac{\frac{4 \sqrt{10}}{15} \pi N S\left(\gamma_{e}-\gamma_{o}\right)}{1-\frac{4}{3} \pi N\langle\alpha\rangle} \\
& n_{e} \approx \frac{7}{2 \sqrt{10}}+\frac{\frac{2 \sqrt{10}}{5} \pi N\langle\alpha\rangle}{1-\frac{4}{3} \pi N\langle\alpha\rangle}-\frac{\frac{2 \sqrt{10}}{15} \pi N S\left(\gamma_{e}-\gamma_{o}\right)}{1-\frac{4}{3} \pi N\langle\alpha\rangle} .
\end{aligned}
$$

Birefringence $\Delta n$ is defined as the difference between the extraordinary and ordinary refractive indices, $\Delta n=n_{e}-n_{o}$ and the average refractive indices $\langle n\rangle$ is defined as $\langle n\rangle=\left(n_{e}+2 n_{o}\right) / 3$. Based on these two definitions, $n_{e}$ and $n_{o}$ can be rewritten as follows [14], [25]:

$$
\begin{aligned}
& n_{e}=\langle n\rangle+\frac{2}{3} \Delta n \\
& n_{o}=\langle n\rangle-\frac{1}{3} \Delta n .
\end{aligned}
$$

By comparing (8) with (9), we find the expressions for $\langle n\rangle$ and $\Delta n$

$$
\begin{aligned}
& \langle n\rangle \approx \frac{7}{2 \sqrt{10}}+\frac{\frac{2 \sqrt{10}}{5} \pi N\langle\alpha\rangle}{1-\frac{4}{3} \pi N\langle\alpha\rangle} \\
& \Delta n=\frac{\frac{2 \sqrt{10}}{5} \pi N S\left(\gamma_{e}-\gamma_{o}\right)}{1-\frac{4}{3} \pi N\langle\alpha\rangle} .
\end{aligned}
$$

Here, $N$ is the molecular packing density (or number of molecules per unit volume) which is equal to $\rho N_{A} / M$; where $\rho$ is the LC density, $N_{A}$ is the Avogadro number, and $\mathrm{M}$ is the molecular weight. The term $4 \pi N\langle\alpha\rangle / 3$ in the denominator of (10) and (11) is much smaller than one and the average polarizability $\langle\alpha\rangle$ is not sensitive to the temperature [24], [30]. Thus, the average refractive index is mainly determined by the LC density while the birefringence by the order parameter S and the LC density. However, (10) and (11) do not disclose the detailed temperature effects on $\langle n\rangle$ and $\Delta n$. To describe the temperature dependent birefringence, Haller approximation has been commonly employed [26]

$$
\Delta n(T)=(\Delta n)_{o}\left(1-\frac{T}{T_{c}}\right)^{\beta} .
$$

In (12), $(\Delta n)_{o}$ is the LC birefringence in the crystalline state (or $\mathrm{T}=0 \mathrm{~K}$ ), the exponent $\beta$ is a material constant, and $T_{c}$ is the clearing temperature of the LC material underinvestigation. From the measured experimental data, we found the average refractive index decreases linearly with increasing temperature as [14]

$$
\langle n\rangle=A-B T
$$

Equation (13) can also be approximated from (10) by assuming that $4 \pi N\langle\alpha\rangle / 3 \ll 1,\langle\alpha\rangle$ is insensitive to the temperature, and density $\rho$ linearly decreases with increasing temperature. Substituting (12) and (13) back to (9a) and (9b), we derive the fourparameter model for describing the temperature dependence of the LC refractive indices [14]

$$
\begin{aligned}
& n_{e}(T) \approx A-B T+\frac{2(\Delta n)_{o}}{3}\left(1-\frac{T}{T_{c}}\right)^{\beta} \\
& n_{o}(T) \approx A-B T-\frac{(\Delta n)_{o}}{3}\left(1-\frac{T}{T_{c}}\right)^{\beta} .
\end{aligned}
$$

Although (14) has four parameters, we can get $[A, B]$ and $\left[(\Delta n)_{o}, \beta\right]$ respectively by two-stage fittings. To obtain $[A, B]$, we fit the average refractive index $\langle n\rangle=\left(n_{e}+2 n_{o}\right) / 3$ as a function of temperature using (13). To find $\left[(\Delta n)_{o}, \beta\right]$, we fit the birefringence data as a function of temperature using (12). Therefore, these two sets of parameters can be obtained separately from the same set of refractive indices but at different forms.

For direct-view and projection LC displays, the LC mixture employed usually exhibits a low birefringence $(\Delta \mathrm{n}<0.1)$ and high clearing temperature $\left(\mathrm{T}_{\mathrm{c}}>90^{\circ} \mathrm{C}\right)$. If the operating temperature is much lower than the clearing temperature, i.e., $T \ll T_{c}$, the $\left(1-T / T_{c}\right)^{\beta}$ term in (14a) and (14b) can be expanded into power series. Keeping the first three terms, we obtain

$$
\begin{aligned}
& n_{e}(T)=A_{e}-B_{e} T-C_{e} T^{2} \\
& n_{o}(T)=A_{o}-B_{o} T+C_{o} T^{2}
\end{aligned}
$$


where $A_{e}=A+\left(2(\Delta n)_{o} / 3\right), B_{e}=B+\left(2(\Delta n)_{o} \beta / 3 T_{c}\right)$, $C_{e}=(\Delta n)_{o} \beta(1-\beta) / 3 T_{c}^{2}, A_{o}=A-\left((\Delta n)_{o} / 3\right), B_{o}=$ $B-\left((\Delta n)_{o} \beta / 3 T_{c}\right)$, and $C_{o}=(\Delta n)_{o} \beta(1-\beta) / 6 T_{c}^{2}$.

Equations (15a) and (15b) indicate that the LC refractive indices have a parabolic relationship with temperature, provided that the operating temperature is far from $T_{c}$. For $n_{e}$, the placket of the parabola is downward and, however, for $n_{o}$ the placket is upward.

From (14), we can derive the temperature gradient for $n_{e}$ and $n_{o}$, respectively [25]

$$
\begin{aligned}
& \frac{d n_{e}}{d T}=-B-\frac{2 \beta(\Delta n)_{o}}{3 T_{c}\left(1-\frac{T}{T_{c}}\right)^{1-\beta}} \\
& \frac{d n_{o}}{d T}=-B+\frac{\beta(\Delta n)_{o}}{3 T_{c}\left(1-\frac{T}{T_{c}}\right)^{1-\beta}} .
\end{aligned}
$$

In (16a), both terms in the right-hand side are negative, independent of temperature. This implies that $n_{e}$ decreases as the temperature increases throughout the entire nematic range. However, (16b) consists of a negative term $(-B)$ and a positive term which depends on the temperature. In the low temperature regime $\left(T \ll T_{c}\right)$, the positive term could be smaller than the negative term resulting in a negative $d n_{o} / d T$. As the temperature increases, the positive term also increases. As $T$ approaches $T_{c}, d n_{o} / d T$ jumps to a large positive number. In the intermediate, there exists a transition temperature where $d n_{o} / d T=0$. We define this temperature as the crossover temperature $T_{o}$ for $n_{o}$. To find $T_{o}$, we simply solve $d n_{o} / d T=0$ from (16b) [25].

\section{EXPERIMENT}

We have selected seven LC mixtures, MLC-9200-000, MLC-9200-100, MLC-6241-000, MLC-6608, TL-216, E44, E7 and two LC compounds (5CB and 5PCH) as examples to validate the physical models we derived. The first five LC mixtures are commonly employed for TFT-LCD applications. MLC-9200-000 and MLC-9200-100 are Merck two-bottle mixtures. These two mixtures have very similar physical properties except for different birefringence. MLC-9200-000 has $\Delta \mathrm{n} \sim 0.085$ and MLC-9200-100 has $\Delta \mathrm{n} \sim 0.114$. By mixing these two bottles in a proper fraction, birefringence in the $0.085-0.114$ range can be formulated. The mixture MLC-6608 has a negative dielectric anisotropy, $\Delta \varepsilon \sim-4.2$. It is a representative mixture for vertical alignment. Lastly, TL-216 is a high birefringence $(\Delta \mathrm{n} \sim 0.213)$ LC mixture used in a color-sequential liquid-crystal-on-silicon (LCoS) projection display [27]. We measured their UV absorption spectra and refractive indices. The UV absorption spectra affect the material's photostability and birefringence. The measured refractive indices are used to validate the abovementioned theories.

\section{A. UV Absorption Spectra}

The UV absorption spectra of MLC-9200-000, MLC-9200100, MLC-6608, and TL-216 were measured at $T=23{ }^{\circ} \mathrm{C}$ by dissolving each LC mixture into a UV transparent LC host MLC-6815. To avoid detector saturation, the guest LC mixture was controlled at $1 \mathrm{wt} \%$. We used the dual channel Cary $500 \mathrm{UV}$

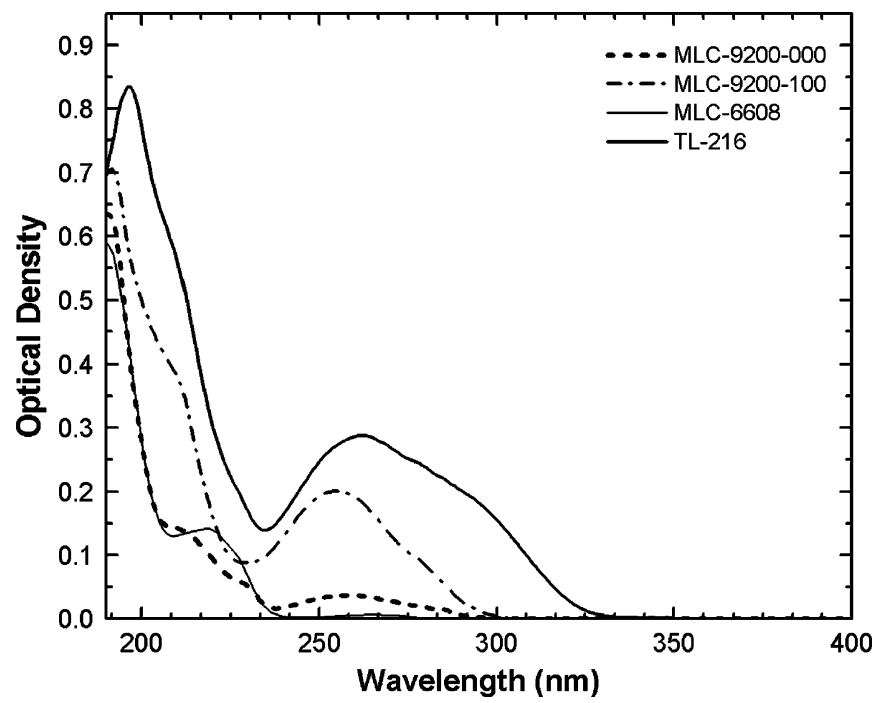

Fig. 1. UV absorption spectrum of $1 \mathrm{wt} \%$ MLC-9200-000, MLC-9200-000, MLC-6608, and TL-216 dissolved in a UV transparent MLC-6815 mixture. Measurement was made at $T=23^{\circ} \mathrm{C}$. The dashed, dashed-dot, black solid, and grey solid lines are UV absorption spectra of MLC-9200-000, MLC-9200-100, MLC-6608, and TL-216, respectively. Cell gap $d=6 \mu \mathrm{m}$.

spectrophotometer and quartz cells for the UV measurements. The quartz substrates were sputtered with $\mathrm{SiO}_{2}$ alignment layers for achieving homogeneous alignment, but no indium-tin-oxide conductive electrode was deposited. The cell gap was controlled at $d=6 \mu \mathrm{m}$.

Fig. 1 depicts the UV absorption spectra of 1 wt \% MLC-9200-000, MLC-9200-100, MLC-6608, and TL-216 dissolved in MLC-6815. The dashed, dashed-dot, black solid line, and grey solid line represent their UV absorption spectra, respectively. In Fig. 1, MLC-9200-100 has a longer resonance absorption wavelength than MLC-9200-000. This explains why MLC-9200-100 $(\Delta n \sim 0.114)$ exhibits a higher birefringence than MLC-9200-000 ( $\Delta n \sim 0.085)$. MLC-9200-000 and MLC-6608 $(\Delta n \sim 0.083)$ have similar resonance absorption peaks so that the two LC mixtures have a comparable birefringence, but their dielectric anisotropy has opposite signs. Among the four mixtures studied, TL-216 has the longest absorption wavelengths. Thus, TL-216 has the highest birefringence among these LC mixtures investigated. However, its UV stability is the worst among these four. UV stability is especially important to LCoS projection display where the ultra-high-pressure lamp emits harmful UV contents. Although UV and blue cutoff wavelength filters are used to remove the harmful UV contents, any residual transmitted UV light could degrade the long-term stability of the LC materials or alignment layers [28]. Whichever fails first will deteriorate the LC panel's overall performance and limit the projector's lifetime.

\section{B. Refractive Indices}

We measured the refractive indices of four low birefringence TFT LC mixtures [MLC-9200-000 $\left(\Delta n \sim 0.085 ; T_{c}=362 \mathrm{~K}\right)$, MLC-9200-100 $\left(\Delta n \sim 0.114 ; T_{c}=363 \mathrm{~K}\right)$, MLC-6608 $\left(\Delta n \sim 0.083 ; T_{c}=363 \mathrm{~K} ; \Delta \varepsilon<0\right)$, and MLC-6241-000 $\left.\left(\Delta n \sim 0.086 ; T_{c}=374 \mathrm{~K}\right)\right]$, three high birefringence LC mixtures [TL-216 $\left(\Delta n \sim 0.213 ; T_{c}=353.3 \mathrm{~K}\right)$, E7 $(\Delta n \sim 0.217$; $\left.T_{c}=331 \mathrm{~K}\right)$, and E44 $\left.\left(\Delta n \sim 0.258 ; T_{c}=373 \mathrm{~K}\right)\right]$, and two 
TABLE I

MEASURED REFRACTIVE INDICES $\left(n_{e}\right.$ AND $n_{o}$ ) OF MLC-9200-000 AT $\lambda=450,486,546,589,633$, AND 656 nm AT DifFERENT TEMPERATURES. THE DATA With an Asterisk are Fitting Values Using the Extended Cauchy Equation (EQ. (2)) Because These Values are Too Small to MEASURE From OUR ABBE REFRACTOMETER

\begin{tabular}{|c|c|c|c|c|c|c|c|c|c|c|c|c|}
\hline \multirow{3}{*}{$\begin{array}{l}\mathrm{T} \\
\left({ }^{\circ} \mathrm{C}\right)\end{array}$} & \multicolumn{12}{|c|}{$\lambda(\mathrm{nm})$} \\
\hline & \multicolumn{2}{|c|}{450} & \multicolumn{2}{|l|}{486} & \multicolumn{2}{|l|}{546} & \multicolumn{2}{|l|}{589} & \multicolumn{2}{|c|}{633} & \multicolumn{2}{|c|}{656} \\
\hline & $n_{e}$ & $n_{o}$ & $n_{e}$ & $n_{o}$ & $n_{e}$ & $n_{o}$ & $n_{e}$ & $n_{o}$ & $n_{e}$ & $n_{o}$ & $n_{e}$ & $n_{o}$ \\
\hline 15 & 1.5821 & 1.4932 & 1.5764 & 1.4884 & 1.5689 & 1.4824 & 1.5657 & 1.4794 & 1.5626 & 1.4771 & 1.5613 & 1.4752 \\
\hline 20 & 1.5788 & 1.4901 & 1.5730 & 1.4861 & 1.5662 & 1.4812 & 1.5625 & 1.478 & 1.5598 & 1.4762 & 1.5584 & 1.4752 \\
\hline 25 & 1.5755 & 1.4885 & 1.5698 & 1.4845 & 1.5634 & 1.4793 & 1.5596 & 1.4767 & 1.5569 & 1.4746 & 1.5553 & 1.4732 \\
\hline 30 & 1.5729 & $1.4882^{*}$ & 1.5669 & $1.4838^{*}$ & 1.5602 & 1.4782 & 1.5565 & 1.4752 & 1.5532 & 1.4727 & 1.5518 & 1.4717 \\
\hline 35 & 1.5683 & $1.4844^{*}$ & 1.5637 & $1.4807^{*}$ & 1.5571 & 1.4762 & 1.5533 & 1.4737 & 1.5507 & 1.4717 & 1.5497 & 1.4708 \\
\hline 40 & 1.5673 & $1.4820^{*}$ & 1.5601 & $1.4784^{*}$ & 1.5536 & 1.4741 & 1.5501 & 1.4716 & 1.5472 & 1.4695 & 1.5458 & 1.4688 \\
\hline 45 & 1.5616 & $1.4786^{*}$ & 1.5565 & $1.4756^{*}$ & 1.5505 & 1.4721 & 1.5466 & 1.4701 & 1.5440 & 1.4680 & 1.5430 & 1.4679 \\
\hline 50 & 1.5594 & $1.4791^{*}$ & 1.5534 & $1.4757^{*}$ & 1.5468 & 1.4715 & 1.5430 & 1.4692 & 1.5407 & 1.4674 & 1.5396 & 1.4665 \\
\hline 55 & 1.5544 & $1.4768^{*}$ & 1.5494 & $1.4736^{*}$ & 1.5430 & 1.4697 & 1.5396 & 1.4675 & 1.5368 & 1.4661 & 1.5352 & 1.4649 \\
\hline
\end{tabular}

TABLE II

MEASURED REFRACTIVE INDICES $\left(n_{e}\right.$ AND $n_{o}$ ) OF MLC-9200-100 AT $\lambda=450,486,546,589,633$, AND 656 nm AT DifFERENT TEMPERATURES. THE DATA With an Asterisk are Fitting Values Using the Extended Cauchy Equation (EQ. (2)) Because These Values are Too Small to MEASURE From OUR ABBE REFRACTOMETER

\begin{tabular}{|c|c|c|c|c|c|c|c|c|c|c|c|c|}
\hline \multirow{3}{*}{$\begin{array}{c}\mathrm{T} \\
\left({ }^{\circ} \mathrm{C}\right)\end{array}$} & \multicolumn{12}{|c|}{$\lambda(\mathrm{nm})$} \\
\hline & \multicolumn{2}{|c|}{450} & \multicolumn{2}{|c|}{486} & \multicolumn{2}{|c|}{546} & \multicolumn{2}{|c|}{589} & \multicolumn{2}{|c|}{633} & \multicolumn{2}{|c|}{656} \\
\hline & $n_{e}$ & $n_{o}$ & $n_{e}$ & $n_{o}$ & $n_{e}$ & $n_{o}$ & $n_{e}$ & $n_{o}$ & $n_{e}$ & $n_{o}$ & $n_{e}$ & $n_{o}$ \\
\hline$\overline{15}$ & 1.6343 & $1.5081^{*}$ & 1.6272 & 1.5038 & 1.6169 & 1.4977 & 1.6112 & 1.4949 & 1.607 & 1.4926 & 1.6056 & 1.4916 \\
\hline 20 & 1.6312 & $1.5057^{*}$ & 1.6233 & 1.5022 & 1.6131 & 1.4962 & 1.6078 & 1.4934 & 1.6039 & 1.4908 & 1.6013 & 1.4900 \\
\hline 25 & 1.6276 & $1.5050^{*}$ & 1.6203 & 1.5005 & 1.6095 & 1.4954 & 1.6041 & 1.4919 & 1.5998 & 1.4895 & 1.5984 & 1.4887 \\
\hline 30 & 1.6251 & $1.5037^{*}$ & 1.6164 & 1.4993 & 1.6059 & 1.4938 & 1.6010 & 1.4908 & 1.5967 & 1.4884 & 1.5951 & 1.4875 \\
\hline 35 & 1.6209 & $1.5015^{*}$ & 1.6126 & 1.4974 & 1.6025 & 1.4924 & 1.5970 & 1.4890 & 1.5931 & 1.4871 & 1.5914 & 1.4858 \\
\hline 40 & 1.6156 & $1.5015^{*}$ & 1.6085 & $1.4969^{*}$ & 1.5988 & 1.4913 & 1.5934 & 1.4879 & 1.5891 & 1.4855 & 1.5878 & 1.4846 \\
\hline 45 & 1.6116 & $1.5002^{*}$ & 1.6052 & $1.4954^{*}$ & 1.5948 & 1.4894 & 1.5897 & 1.4864 & 1.5854 & 1.4836 & 1.5838 & 1.4825 \\
\hline 50 & 1.6072 & $1.4994^{*}$ & 1.6006 & $1.4945^{*}$ & 1.5905 & 1.4884 & 1.5854 & 1.4853 & 1.5815 & 1.4825 & 1.5797 & 1.4813 \\
\hline 55 & 1.6024 & $1.4974^{*}$ & 1.5961 & $1.4929^{*}$ & 1.5861 & 1.4872 & 1.5811 & 1.4842 & 1.5770 & 1.4816 & 1.5756 & 1.4806 \\
\hline
\end{tabular}

LC compounds [5CB $\left(\Delta n \sim 0.177 ; T_{c}=308.3 \mathrm{~K}\right)$ and $5 \mathrm{PCH}$ $\left.\left(\Delta n \sim 0112 ; T_{c}=328 \mathrm{~K}\right)\right]$ using a Multi-wavelength Abbe refractometer (Atago DR-M4) at $\lambda=450,486,546,589,633$, and $656 \mathrm{~nm}$. The accuracy of the Abbe refractometer is up to the fourth decimal. For a given wavelength, we measured the refractive indices of these LCs from $15^{\circ} \mathrm{C}$ to $50{ }^{\circ} \mathrm{C}$ with $5{ }^{\circ} \mathrm{C}$ interval. The temperature of the Abbe refractometer is controlled by a circulating constant temperature bath (Atago Model 60-C3). The LC molecules are aligned perpendicular to the main and secondary prism surfaces of the Abbe refractometer by coating these two surfaces with a surfactant comprising of 0.294 wt \% hexadecyletri-methyle-ammonium bromide (HMAB) in methanol solution. Both $n_{e}$ and $n_{o}$ are obtained through a polarizing eyepiece. The measured data of MLC-9200-000, MLC-9200-100, MLC-6608, TL-216, E44 and E7 are listed in Tables I-VI, respectively. The data with an asterisk in the top right corner stand for the calculated values using the extended Cauchy equations since these values are too small to measure using our Abbe refractometer.

\section{RESULTS AND DISCUSSIONS}

In this section, we will use the experimental data of four LC materials to validate the models we derived in Section II. We will discuss the wavelength effect and temperature effect, respectively.

\section{A. Wavelength Effect}

Fig. 2 depicts the wavelength-dependent refractive indices of MLC-9200-000 and MLC-9200-100 at $T=25{ }^{\circ} \mathrm{C}$. The open and filled circles represent the experimental data of $n_{e}$ and $n_{o}$ for MLC-9200-000, and the open and filled triangles are the corresponding $n_{e}$ and $n_{o}$ for MLC-9200-100, respectively. The solid and dashed lines are fittings using the three-coefficient ((2)) and two-coefficient Cauchy model [see (3)], respectively. Table VII lists the fitting parameters. In Fig. 2, all the solid and dashed lines fit the experimental data very well and the dashed lines almost completely overlap with the solid lines. It means the three- and two-coefficient Cauchy models fit 
TABLE III

MEASURED REFRACTIVE INDices $\left(n_{e}\right.$ AND $n_{o}$ ) OF MLC-6608 AT $\lambda=450,486,546,589,633$, AND 656 nm AT DifFERENT TEMPERATURE. THE DATA With an Asterisk are Fitting Values Using the Extended Cauchy Equation (EQ. (2)) Because These Values are Too Small to MEASURE FROM OUR ABBE REFRACTOMETER

\begin{tabular}{|c|c|c|c|c|c|c|c|c|c|c|c|c|}
\hline \multirow{3}{*}{$\begin{array}{c}\mathrm{T} \\
\left({ }^{\circ} \mathrm{C}\right)\end{array}$} & \multicolumn{12}{|c|}{$\lambda(\mathrm{nm})$} \\
\hline & \multicolumn{2}{|r|}{450} & \multicolumn{2}{|c|}{486} & \multicolumn{2}{|c|}{546} & \multicolumn{2}{|c|}{589} & \multicolumn{2}{|c|}{633} & \multicolumn{2}{|c|}{656} \\
\hline & $n_{e}$ & $n_{o}$ & $n_{e}$ & $n_{o}$ & $n_{e}$ & $n_{o}$ & $n_{e}$ & $n_{o}$ & $n_{e}$ & $n_{o}$ & $n_{e}$ & $n_{o}$ \\
\hline$\overline{15}$ & 1.5766 & $1.4887^{*}$ & 1.5717 & $1.4852^{*}$ & 1.5654 & 1.4808 & 1.5621 & 1.4785 & 1.5592 & 1.4763 & 1.5581 & 1.4758 \\
\hline 20 & 1.5733 & $1.4859^{*}$ & 1.5687 & $1.4828^{*}$ & 1.5622 & 1.4789 & 1.5595 & 1.4768 & 1.5565 & 1.4751 & 1.5554 & 1.4745 \\
\hline 25 & 1.5704 & $1.4854^{*}$ & 1.5655 & $1.4819^{*}$ & 1.5595 & 1.4775 & 1.5562 & 1.4753 & 1.5531 & 1.4731 & 1.5522 & 1.4725 \\
\hline 30 & 1.5678 & $1.4826^{*}$ & 1.5628 & $1.4794^{*}$ & 1.5564 & 1.4754 & 1.553 & 1.4733 & 1.5504 & 1.4714 & 1.5495 & 1.4708 \\
\hline 35 & 1.5645 & $1.4823^{*}$ & 1.5598 & $1.4787^{*}$ & 1.5534 & 1.4742 & 1.5500 & 1.4718 & 1.5473 & 1.4696 & 1.5466 & 1.469 \\
\hline 40 & 1.5612 & $1.4782^{*}$ & 1.5558 & $1.4753^{*}$ & 1.5500 & 1.4718 & 1.5468 & 1.4699 & 1.5441 & 1.4681 & 1.5433 & 1.4677 \\
\hline 45 & 1.5577 & $1.4783^{*}$ & 1.553 & $1.4749^{*}$ & 1.5467 & 1.4707 & 1.5436 & 1.4685 & 1.5407 & 1.4664 & 1.5399 & 1.4659 \\
\hline 50 & 1.5543 & $1.4755^{*}$ & 1.5494 & $1.4725^{*}$ & 1.5432 & 1.4688 & 1.5399 & 1.4668 & 1.5375 & 1.4653 & 1.5362 & 1.4644 \\
\hline 55 & 1.5498 & $1.4734^{*}$ & 1.5453 & $1.4706^{*}$ & 1.5396 & 1.4672 & 1.5361 & 1.4653 & 1.5338 & 1.4637 & 1.533 & 1.4631 \\
\hline
\end{tabular}

TABLE IV

MEASURED REFRACTIVE INDICES $\left(n_{e}\right.$ AND $n_{o}$ ) OF TL-216 AT $\lambda=450,486,546,589,633$, AND $656 \mathrm{~nm}$ AT DifFERENT TEMPERATURE

\begin{tabular}{|c|c|c|c|c|c|c|c|c|c|c|c|c|}
\hline \multirow{3}{*}{$\begin{array}{c}\mathrm{T} \\
\left({ }^{\circ} \mathrm{C}\right)\end{array}$} & \multicolumn{12}{|c|}{$\lambda(\mathrm{nm})$} \\
\hline & \multicolumn{2}{|c|}{450} & \multicolumn{2}{|c|}{486} & \multicolumn{2}{|c|}{546} & \multicolumn{2}{|c|}{589} & \multicolumn{2}{|c|}{633} & \multicolumn{2}{|c|}{656} \\
\hline & $n_{e}$ & $n_{o}$ & $n_{e}$ & $n_{o}$ & $n_{e}$ & $n_{o}$ & $n_{e}$ & $n_{o}$ & $n_{e}$ & $n_{o}$ & $n_{e}$ & $n_{o}$ \\
\hline$\overline{15}$ & 1.7910 & 1.5430 & 1.7730 & 1.5365 & 1.7520 & 1.5290 & 1.7408 & 1.5246 & 1.7328 & 1.5212 & 1.7292 & 1.5204 \\
\hline 20 & 1.7851 & 1.5408 & 1.7677 & 1.5349 & 1.7472 & 1.5276 & 1.7365 & 1.5240 & 1.7288 & 1.5203 & 1.7245 & 1.5190 \\
\hline 25 & 1.7806 & 1.5403 & 1.7623 & 1.5340 & 1.7412 & 1.5268 & 1.7315 & 1.5228 & 1.7235 & 1.5192 & 1.7184 & 1.5174 \\
\hline 30 & 1.7753 & 1.5400 & 1.7570 & 1.5334 & 1.7361 & 1.5254 & 1.7259 & 1.5218 & 1.7186 & 1.5185 & 1.7145 & 1.5170 \\
\hline 35 & 1.7696 & 1.5401 & 1.7506 & 1.5332 & 1.7312 & 1.525 & 1.7211 & 1.5209 & 1.7136 & 1.5175 & 1.7094 & 1.5161 \\
\hline 40 & 1.7640 & 1.5381 & 1.7448 & 1.5318 & 1.7251 & 1.5242 & 1.7156 & 1.5204 & 1.7081 & 1.5170 & 1.7045 & 1.5158 \\
\hline 45 & 1.7566 & 1.5368 & 1.7388 & 1.5309 & 1.7190 & 1.5238 & 1.7093 & 1.5198 & 1.7021 & 1.5163 & 1.6987 & 1.5155 \\
\hline 50 & 1.7497 & 1.5375 & 1.7321 & 1.5310 & 1.7127 & 1.5234 & 1.7030 & 1.5193 & 1.6959 & 1.5158 & 1.6925 & 1.5149 \\
\hline 55 & 1.7413 & 1.5379 & 1.7247 & 1.5314 & 1.7048 & 1.5232 & 1.6957 & 1.5196 & 1.6891 & 1.5159 & 1.6861 & 1.5143 \\
\hline
\end{tabular}

the experimental data of MLC-9200-000 and MLC-9200-100 equally well. From Table VII, we find the fitting Cauchy coefficients $C_{e}$ and $C_{o}$ are considerably smaller than the $B_{e}$ and $B_{o}$ for both MLC-9200-000 and MLC-9200-100. This indicates that the magnitude of the $\lambda^{-4}$ term is much smaller than that of the $\lambda^{-2}$ term and can be ignored. For low birefringence LC materials, the two-coefficient Cauchy model works equally well as the three-coefficient one.

Fig. 3 depicts the wavelength-dependent refractive indices of MLC-6608 and TL-216 at $T=25^{\circ} \mathrm{C}$. MLC-6608 is a negative LC mixture; its dielectric anisotropy is $\Delta \varepsilon=-4.2$. The open and filled circles and triangles represent the experimental data of $n_{e}$ and $n_{o}$ for MLC-6608 and TL-216, respectively. The solid and dashed lines are fittings using the three-coefficient $((2))$ and two-coefficient Cauchy models ((3)). The fitting parameters are listed in Table VII. In Fig. 3, the dashed lines almost completely overlap with the solid lines; both models fit the experimental data of MLC-6608 very well. However, there are some differences between the fitting curves using the three- and two-coef- ficient models for TL-216. The fitting error of the three-coefficient Cauchy model $\left(\chi^{2} \sim 10^{-7}\right)$ is one order smaller than that of the two-coefficient Cauchy model $\left(\chi^{2} \sim 10^{-6}\right)$. Here, the $\chi^{2}$ deviation is defined as the sum of the squares of observed values minus expected values and then divided by the expected values. In Table VII, the Cauchy coefficients $C_{e}$ and $C_{o}$ are considerably smaller than $B_{e}$ and $B_{o}$ for MLC-6608, however, the coefficient $C_{e}$ is comparable to $B_{e}$ for TL-216. This indicates that both the three- and two-coefficient Cauchy models fit the experimental data of MLC-6608 well, but the three-coefficient Cauchy model fits the $n_{e}$ data better than the two-coefficient Cauchy model for TL-216. Thus, for TL-216 the $\lambda^{-4}$ term is comparable to the $\lambda^{-2}$ term and cannot be ignored.

In Fig. 4, we show the wavelength-dependent refractive indices of E44, E7, 5CB, 5PCH, and MLC-6241-000 at $\mathrm{T}=25^{\circ} \mathrm{C}$. The birefringence of E44, E7, 5CB, 5PCH and MLC-6241-000 is $\sim 0.258,0.217,0.177,0.116$, and 0.086, respectively, at $\lambda=589 \mathrm{~nm}$ and $\mathrm{T}=25^{\circ} \mathrm{C}$. The open and filled pentagons, circles, squares, upward- and downward-triangles 
TABLE V

MEASURED REFRACTIVE INDICES $\left(n_{e}\right.$ AND $n_{o}$ ) OF E44 AT $\lambda=450,486,546,589,633$, AND 656 nm AT DiFFERENT TEMPERATURES

\begin{tabular}{|c|c|c|c|c|c|c|c|c|c|c|c|c|}
\hline \multirow{3}{*}{$\begin{array}{c}\mathrm{T} \\
\left({ }^{\circ} \mathrm{C}\right)\end{array}$} & \multicolumn{12}{|c|}{$\lambda(\mathrm{nm})$} \\
\hline & \multicolumn{2}{|c|}{450} & \multicolumn{2}{|c|}{486} & \multicolumn{2}{|c|}{546} & \multicolumn{2}{|c|}{589} & \multicolumn{2}{|c|}{633} & \multicolumn{2}{|c|}{656} \\
\hline & $n_{e}$ & $n_{o}$ & $n_{e}$ & $n_{o}$ & $n_{e}$ & $n_{o}$ & $n_{e}$ & $n_{o}$ & $n_{e}$ & $n_{o}$ & $n_{e}$ & $n_{o}$ \\
\hline 15 & 1.8652 & 1.5509 & 1.8393 & 1.5429 & 1.8102 & 1.5338 & 1.7960 & 1.5295 & 1.7856 & 1.5259 & 1.7807 & 1.5245 \\
\hline 20 & 1.8607 & 1.5485 & 1.8339 & 1.5415 & 1.8048 & 1.5330 & 1.7906 & 1.5285 & 1.7806 & 1.5245 & 1.7760 & 1.5233 \\
\hline 25 & 1.8539 & 1.5478 & 1.8279 & 1.5408 & 1.7993 & 1.5322 & 1.7855 & 1.5275 & 1.7754 & 1.5242 & 1.7709 & 1.5221 \\
\hline 30 & 1.848 & 1.5491 & 1.8221 & 1.5407 & 1.7934 & 1.5315 & 1.7800 & 1.5269 & 1.7699 & 1.5233 & 1.7653 & 1.5222 \\
\hline 35 & 1.8408 & 1.5464 & 1.8155 & 1.5393 & 1.7874 & 1.5308 & 1.7742 & 1.5262 & 1.7643 & 1.5226 & 1.7595 & 1.5213 \\
\hline 40 & 1.8338 & 1.5474 & 1.8089 & 1.5391 & 1.7809 & 1.5296 & 1.7679 & 1.5251 & 1.7579 & 1.5217 & 1.7533 & 1.5198 \\
\hline 45 & 1.8262 & 1.5464 & 1.8018 & 1.5389 & 1.7747 & 1.5298 & 1.7614 & 1.5250 & 1.7516 & 1.5208 & 1.7473 & 1.5196 \\
\hline 50 & 1.8192 & 1.5468 & 1.7948 & 1.5384 & 1.7679 & 1.5291 & 1.7547 & 1.5246 & 1.7454 & 1.5211 & 1.7410 & 1.5198 \\
\hline 55 & 1.8111 & 1.5464 & 1.7871 & 1.5384 & 1.7604 & 1.5289 & 1.7480 & 1.5244 & 1.7384 & 1.5202 & 1.7343 & 1.5189 \\
\hline
\end{tabular}

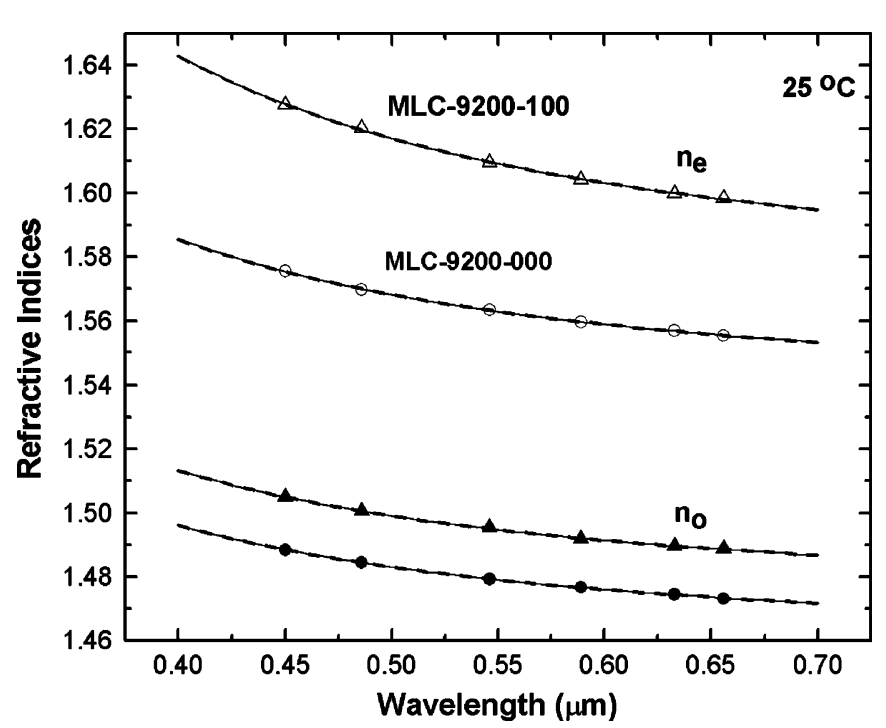

Fig. 2. Wavelength-dependent refractive indices of MLC-9200-000 and MLC-9200-100 at $T=25{ }^{\circ} \mathrm{C}$. Open and filled circles and triangles, respectively, represent the experimental data for $n_{e}$ and $n_{o}$ of MLC-9200-000 and MLC-9200-100. Solid and dashed lines are fittings by using the three-coefficient [see (2)] and the two-coefficient [see (3)] Cauchy models, respectively. The fitting parameters are listed in Table VII.

are the $n_{e}$ and $n_{o}$ of E44, E7, 5CB, 5PCH and MLC-6241-000, respectively. The solid and dashed lines are fittings using the three-coefficient ((2)) and two-coefficient ((3)) Cauchy models. The fitting parameters are listed in Table VII. From Fig. 4, the three-coefficient Cauchy model fits the experimental $n_{e}$ and $n_{o}$ data for all five LCs well. However, the two-coefficient Cauchy model does not fit the experimental results of E44, E7, and 5CB so nicely as the three-coefficient Cauchy model because these three LCs have a higher birefringence. For 5PCH $(\Delta \mathrm{n} \sim 0.1158)$, the two-coefficient Cauchy model fits the experimental $n_{e}$ and $n_{o}$ data fairly well although the three-coefficient Cauchy model has slightly better fitting. For MLC-6241-000, the fittings by the three- and two-coefficient Cauchy models overlap each other, similar to the other low birefringence LCs. From Figs. 2, 3, and 4, we found a boundary

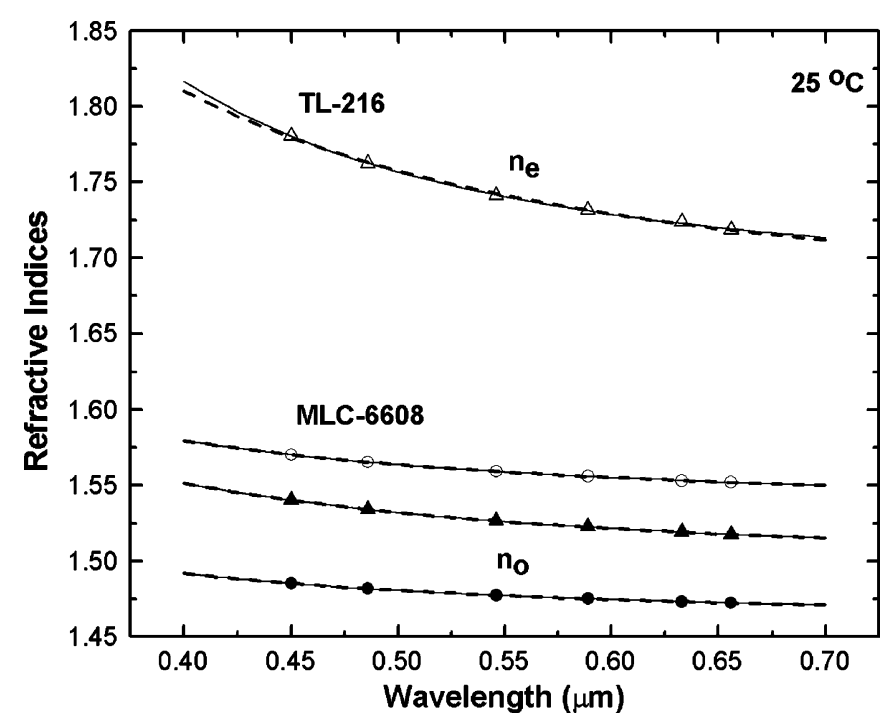

Fig. 3. Wavelength-dependent refractive indices of MLC-6608 and TL-216 at $T=25^{\circ} \mathrm{C}$. Open and filled circles and triangles represent the experimental data of $n_{e}$ and $n_{o}$ for MLC-6608 and TL-216, respectively. While solid and dashed lines are fittings using the three-coefficient [see (2)] and two-coefficient [see (3)] Cauchy models, respectively. The fitting parameters are listed in Table VII.

value of birefringence $(\Delta n=0.12)$ below which the threeand two-coefficient Cauchy models fit the LC refractive indices $n_{e}$ and $n_{o}$ equally well. However, for a high birefringence $(\Delta n>0.12)$ LC the three-coefficient Cauchy model must be used.

In summary, the two-coefficient Cauchy model can be used for describing the wavelength effect of low birefringence $(\Delta n<0.12)$ LC mixtures, no matter they are positive or negative $\Delta \varepsilon$ materials. Since the two-coefficient model has only two parameters, it is more convenient to fit with experimental data. However, for a LC mixture with high birefringence $(\Delta n>0.12)$, the three-coefficient Cauchy model should be used because the $\lambda^{-4}$ term is comparable to the $\lambda^{-2}$ term and cannot be neglected. Based on our experimental results of a dozen LC compounds and mixtures, $\Delta n \sim 0.12$ can be treated as the division between the two-coefficient and three 
TABLE VI

MeAsured ReFractive Indices $\left(n_{e}\right.$ AND $n_{o}$ ) OF E7 AT $\lambda=450,486,546,589,633$, AND $656 \mathrm{~nm}$ AT DifFERENT TEMPERATURE

\begin{tabular}{|c|c|c|c|c|c|c|c|c|c|c|c|c|}
\hline \multirow{3}{*}{$\begin{array}{c}\mathrm{T} \\
\left({ }^{\circ} \mathrm{C}\right)\end{array}$} & \multicolumn{12}{|c|}{$\lambda(\mathrm{nm})$} \\
\hline & \multicolumn{2}{|c|}{450} & \multicolumn{2}{|c|}{486} & \multicolumn{2}{|c|}{546} & \multicolumn{2}{|c|}{589} & \multicolumn{2}{|c|}{633} & \multicolumn{2}{|c|}{656} \\
\hline & $n_{e}$ & $n_{o}$ & $n_{e}$ & $n_{o}$ & $n_{e}$ & $n_{o}$ & $n_{e}$ & $n_{o}$ & $n_{e}$ & $n_{o}$ & $n_{e}$ & $n_{o}$ \\
\hline 15 & 1.817 & 1.5435 & 1.7921 & 1.536 & 1.7664 & 1.5273 & 1.7542 & 1.523 & 1.7446 & 1.5197 & 1.7407 & 1.5179 \\
\hline 20 & 1.8084 & 1.5431 & 1.7847 & 1.5357 & 1.7589 & 1.5269 & 1.7466 & 1.5227 & 1.7378 & 1.5188 & 1.7338 & 1.5177 \\
\hline 25 & 1.8005 & 1.5424 & 1.7763 & 1.5353 & 1.7512 & 1.5268 & 1.7394 & 1.5225 & 1.7305 & 1.5189 & 1.7263 & 1.5176 \\
\hline 30 & 1.7909 & 1.543 & 1.7679 & 1.5357 & 1.7433 & 1.5271 & 1.7317 & 1.5226 & 1.7229 & 1.5189 & 1.719 & 1.5177 \\
\hline 35 & 1.7811 & 1.5448 & 1.7581 & 1.5369 & 1.7344 & 1.5277 & 1.7231 & 1.5231 & 1.7142 & 1.5191 & 1.7102 & 1.5179 \\
\hline 40 & 1.7695 & 1.547 & 1.7472 & 1.5383 & 1.7237 & 1.5287 & 1.7124 & 1.5239 & 1.7037 & 1.5205 & 1.7001 & 1.5189 \\
\hline 45 & 1.7549 & 1.5491 & 1.7333 & 1.5406 & 1.7109 & 1.5308 & 1.7001 & 1.5261 & 1.6919 & 1.5221 & 1.6882 & 1.5206 \\
\hline 50 & 1.7355 & 1.5538 & 1.7154 & 1.5449 & 1.6941 & 1.535 & 1.6837 & 1.5299 & 1.6761 & 1.526 & 1.6721 & 1.5246 \\
\hline 55 & 1.6936 & 1.569 & 1.6779 & 1.5588 & 1.6601 & 1.5479 & 1.6511 & 1.5428 & 1.644 & 1.5377 & 1.6405 & 1.5353 \\
\hline
\end{tabular}

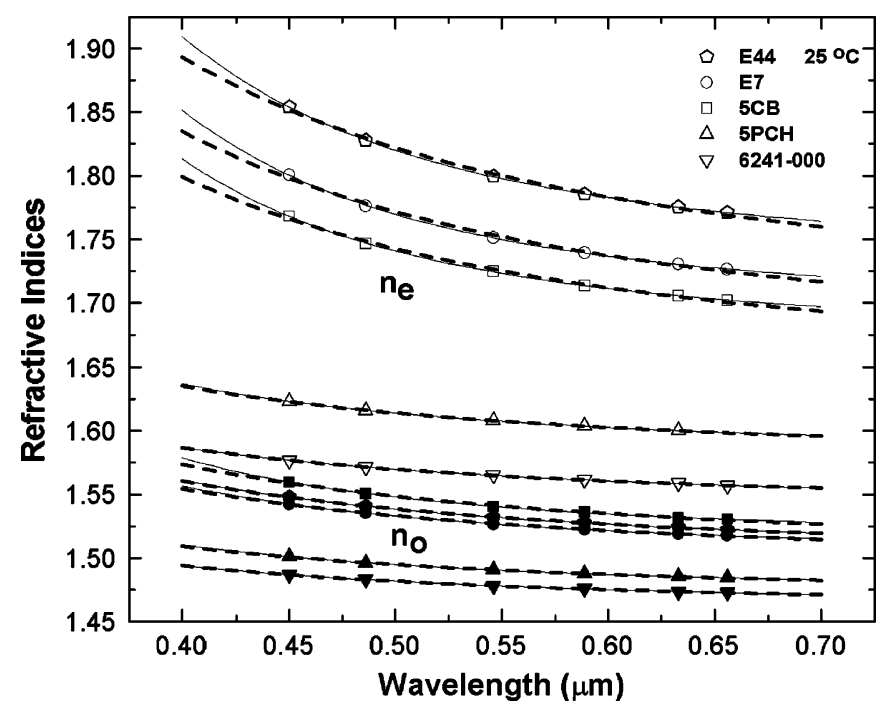

Fig. 4. Wavelength-dependent refractive indices of E44, E7, 5CB, 5PCH, and MLC-6241-000 at $T=25{ }^{\circ} \mathrm{C}$. The open and filled pentagons, circles, triangles, upward- and downward-triangles represent the experimental data of $n_{e}$ and $n_{o}$ for E44, E7, 5CB, 5PCH, and MLC-6241-000, respectively. While solid and dashed lines are fittings using the three-coefficient [see (2)] and two-coefficient [see (3)] Cauchy models, respectively. The fitting parameters are listed in Table VII.

-coefficient Cauchy models. In addition, using the extended Cauchy model we can extrapolate the infrared refractive indices based on the data measured in the visible spectral region. The extrapolated results agree well with those measured directly [29].

\section{B. Temperature Effect}

Equation (14) describes the temperature-dependent refractive indices. Although the four-parameter model has four unknowns: $A, B,(\Delta n)_{o}$, and $\beta$, they can be obtained by the two-step fitting processes. In experiment, we measured the $n_{e}$ and $n_{o}$ using Abbe Refractometer at six wavelengths in the temperature range from 15 to $55^{\circ} \mathrm{C}$. Once the refractive indices are measured, birefringence and average refractive index can be calculated. The first step is to use (13) to fit the average refractive index data and

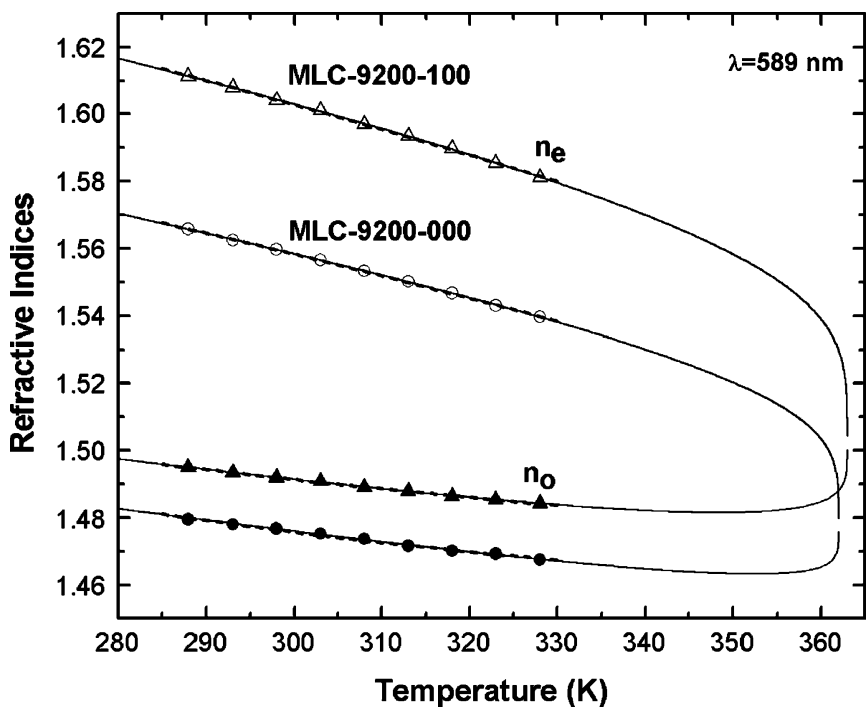

Fig. 5. Temperature-dependent refractive indices of MLC-9200-000 and MLC-9200-100 at $\lambda=589 \mathrm{~nm}$. Open and filled circles and triangles, respectively, represent experimental data of $n_{e}$ and $n_{o}$ for MLC-9200-000 and MLC-9200-100. Solid and dashed lines are fittings using the four-parameter [see (14)] and the three-parameter parabolic [see (15)] models, respectively. The fitting parameters for the two models are listed in Tables VIII and IX, respectively.

obtain parameters $A$ and $B$. The second step is to use (12) to fit the birefringence data and extract $(\Delta n)_{o}$ and $\beta$. Finally, we plug these two sets of parameters into equations. (14a) and (14b) to calculate the temperature-dependent refractive indices. The calculated results are then used to compare with the measured ones. We use the four LC mixtures: MLC-9200-000, MLC-9200-100, MLC-6608, and TL-216 as examples to validate the four-parameter models. At room temperature and $\lambda=589 \mathrm{~nm}$, the birefringence of MLC-9200-000, MLC-9200-100, MLC-6608, and TL-216 is $0.085,0.114,0.083$, and 0.213 , and their clearing temperatures are $362,363,363$, and $353.3 \mathrm{~K}$, respectively. Excellent agreement is obtained between the calculated results and the experimental data.

Fig. 5 depicts the temperature-dependent refractive indices of MLC-9200-000 and MLC-9200-100 at $\lambda=589 \mathrm{~nm}$. The 
TABLE VII

FitTING PARAMETERS OF MLC-9200-000, MLC-9200-100, MLC-6608, TL-216, E44, E7, 5CB, 5PCH, AND MLC-6241-000 FOR THE THREE- AND

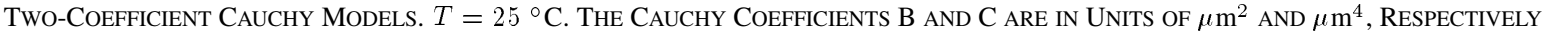

\begin{tabular}{|c|c|c|c|c|c|c|c|}
\hline \multirow[t]{2}{*}{ Materials } & \multirow[t]{2}{*}{ Cauchy Models } & \multicolumn{3}{|c|}{$n_{e}$} & \multicolumn{3}{|c|}{$n_{o}$} \\
\hline & & $A_{e}$ & $B_{e}$ & $C_{e}$ & $A_{o}$ & $B_{o}$ & $C_{o}$ \\
\hline \multirow[t]{2}{*}{ MLC-9200-000 } & gefficient & 1.5382 & 0.0073 & $4.00 \times 10^{-5}$ & 1.4600 & 0.0058 & $3.95 \times 10^{-17}$ \\
\hline & Two-c & 1.5377 & 0.0076 & & 1.4600 & 0.0058 & \\
\hline \multirow[t]{2}{*}{ MLC-9200-100 } & sefficient & 1.5720 & 0.0111 & $4.00 \times 10^{-5}$ & 1.4740 & 0.0063 & $6.04 \times 10^{-18}$ \\
\hline & Two-coefficient & 1.5715 & 0.0114 & & 1.4740 & 0.0063 & \\
\hline \multirow[t]{2}{*}{ MLC-6608 } & Three-coefficient & 1.5359 & 0.0070 & $4.02 \times 10^{-17}$ & 1.4609 & 0.0050 & $3.29 \times 10^{-17}$ \\
\hline & Two-coefficient & 1.5359 & 0.0070 & & 1.4609 & 0.0050 & \\
\hline \multirow[t]{2}{*}{ TL-216 } & Three-coefficient & 1.6772 & 0.0155 & 0.0011 & 1.4989 & 0.0079 & 0.0001 \\
\hline & Two-coefficient & 1.6640 & 0.0234 & & 1.4976 & 0.0086 & \\
\hline \multirow[t]{2}{*}{ E44 } & Three-coefficient & 1.7282 & 0.0121 & 0.0027 & 1.5006 & 0.0091 & 0.0001 \\
\hline & Two-coefficient & 1.6956 & 0.0316 & & 1.4995 & 0.0098 & \\
\hline \multirow[t]{2}{*}{ E7 } & Three-coefficient & 1.6933 & 0.0078 & 0.0028 & 1.4990 & 0.0072 & 0.0003 \\
\hline & Two-coefficient & 1.6594 & 0.0281 & & 1.4952 & 0.0095 & \\
\hline \multirow[t]{2}{*}{$5 \mathrm{CB}$} & Three-coefficient & 1.6708 & 0.0081 & 0.0024 & 1.5139 & 0.0052 & 0.0008 \\
\hline & Two-coefficient & 1.6423 & 0.0251 & & 1.5039 & 0.0112 & \\
\hline \multirow[t]{2}{*}{$5 \mathrm{PCH}$} & Three-coefficient & 1.5801 & 0.0073 & 0.0003 & 1.4718 & 0.0049 & 0.0002 \\
\hline & Two-coefficient & 1.5766 & 0.0094 & & 1.4695 & 0.0064 & \\
\hline \multirow[t]{2}{*}{ MLC-6241-000 } & Three-coefficient & 1.5397 & 0.0075 & $1.03 \times 10^{-5}$ & 1.4601 & 0.0054 & $3.15 \times 10^{-17}$ \\
\hline & Two-coefficient & 1.5395 & 0.0076 & & 1.4601 & 0.0054 & \\
\hline
\end{tabular}

TABLE VIII

FitTing PARAmeters FOR the AVERAge ReFractive IndeX $\langle n\rangle$ (EQ. (13)) AND BIREFRINGENCE $\Delta n$ (EQ. (12)) OF MLC-9200-000, MLC-9200-100, MLC-6608, AND TL-216 AT $\lambda=589 \mathrm{~nm}$

\begin{tabular}{|c|c|c|c|c|}
\hline \multirow[t]{2}{*}{ LCs } & \multicolumn{2}{|c|}{$<n>$} & \multicolumn{2}{|c|}{$\Delta n$} \\
\hline & $A$ & $B\left(\mathrm{~K}^{-1}\right)$ & $(\Delta \mathrm{n})_{\mathrm{o}}$ & $\beta$ \\
\hline MLC-9200-000 & 1.6303 & $4.23 \times 10^{-4}$ & 0.1224 & 0.2246 \\
\hline MLC-9200-100 & 1.6572 & $4.29 \times 10^{-4}$ & 0.1689 & 0.2373 \\
\hline MLC-6608 & 1.6325 & $4.38 \times 10^{-4}$ & 0.1177 & 0.2161 \\
\hline TL-216 & 1.7309 & $4.65 \times 10^{-4}$ & 0.3105 & 0.2143 \\
\hline
\end{tabular}

open and filled circles and triangles are the measured $n_{e}$ and $n_{o}$ for MLC-9200-000 and MLC-9200-100, respectively. The solid and dashed lines are fittings using the four-parameter model ((14)) and three-parameter parabolic model ((15)), respectively. The fitting parameters for these two models are listed in Table VIII and Table IX, respectively. As shown in Fig. 5, the four-parameter model fits the experimental data of MLC-9200-000 and MLC-9200-100 very well in the whole nematic range. The three-parameter parabolic model fits the experimental data of MLC-9200-000 and MLC-9200-100 equally well when the temperature is not too close to the clearing point. This is because both MLC-9200-000 and MLC-9200-100 have a relatively low birefringence while having a high clearing temperature.

Fig. 6 depicts the temperature-dependent refractive indices of MLC-6608 and TL-216 at $\lambda=589 \mathrm{~nm}$. The open and filled circles and triangles are experimental data for $n_{e}$ and $n_{o}$ of MLC-6608 and TL-216, respectively. The solid and dashed lines are fittings using the four-parameter model ((14)) and the threeparameter parabolic model ((15)), respectively. Table VIII and Table IX list the fitting parameters using these two models. In Fig. 6, the four-parameter model fits the experimental data of MLC-6608 and TL-216 very well in the whole nematic range.
The three-parameter parabolic model fits the experimental data of MLC-6608 equally well when the temperature is not too close to the clearing temperature. However, there is still a noticeable difference between the four- and three-parameter models for TL-216. To explain this discrepancy, we need to review the assumption made during the derivation of (15a) and (15b). The two assumptions made there are: 1) low LC birefringence, and 2) $T \ll T_{c}$. Under these conditions, the four-parameter model can be expanded into power series that leads to the three-parameter parabolic model. However, the birefringence of TL-216 is relatively high and the clearing temperature is relatively low so that the power expansion is less accurate. Consequently, the fitting results for TL-216 are less satisfactory. Thus, the three-parameter parabolic model is more suitable for low birefringence LC materials with operating temperature much lower than $T_{c}$.

Fig. 7 depicts the temperature dependant $-d n_{e} / d T$ [see (16a)] and $d n_{o} / d T$ [see (16b)] for MLC-9200-000 and TL-216. The black and grey solid lines represent the calculated $d n_{o} / d T$ curves for MLC-9200-000 and TL-216, respectively, while the dashed lines represent the calculated $-d n_{e} / d T$ curves. The crossover temperatures of MLC-9200-000 and TL-216 are around 80.1 and $52.7{ }^{\circ} \mathrm{C}$, respectively. In Fig. 7, we find the $-d n_{e} / d T$ for both LC mixtures remain positive throughout their nematic range. That means $n_{e}$, the extraordinary refractive index decreases monotonously as the temperature increases in the entire nematic range. However, $d n_{o} / d T$ changes sign at the crossover temperature $T_{o}$ [25]. The $d n_{o} / d T$ is negative when the temperature is below $T_{o}$ whereas becomes positive when the temperature is beyond $T_{o}$. This implies that $n_{o}$, the ordinary refractive index, decreases as temperature increases when the temperature is below $T_{o}$ but increases with temperature when the temperature is above $T_{o}$. This tendency is clearly observed from Figs. 5 and 6. As the temperature approaches the clearing temperature, both $-d n_{e} / d T$ and $d n_{o} / d T$ increase dramatically as depicted in Fig. 7. We also calculated the crossover temperatures of MLC-9200-100 and MLC-6608 and results are found to be $\sim 75^{\circ} \mathrm{C}$ and $81.7^{\circ} \mathrm{C}$, respectively. 
TABLE IX

Fitting Parameters for the three-Parameter Parabolic Model (EQ. (15)). $\lambda=589 \mathrm{~nm}$. The LC Mixtures StUdied ARE MLC-9200-000, MLC-9200-100, MLC-6608, AND TL-216

\begin{tabular}{lrrrrrrr}
\hline LCs & \multicolumn{3}{c}{$n_{e}$} & & \multicolumn{3}{c}{$n_{o}$} \\
\cline { 2 - 3 } \cline { 6 - 8 } & $A_{e}$ & $B_{e}\left(\mathrm{~K}^{-1}\right)$ & $C_{e}\left(\mathrm{~K}^{-2}\right)$ & & $A_{o}$ & $B_{o}\left(\mathrm{~K}^{-1}\right)$ & $C_{o}\left(\mathrm{~K}^{-2}\right)$ \\
\hline MLC-9200-000 & 1.6550 & $1.00 \times 10^{-5}$ & $1.04 \times 10^{-6}$ & & 1.61352 & $6.00 \times 10^{-4}$ & $4.76 \times 10^{-7}$ \\
MLC-9200-100 & 1.7274 & $1.00 \times 10^{-4}$ & $1.05 \times 10^{-6}$ & & 1.6602 & $8.40 \times 10^{-4}$ & $9.26 \times 10^{-7}$ \\
MLC-6608 & 1.6650 & $1.00 \times 10^{-4}$ & $8.89 \times 10^{-7}$ & & 1.6185 & $6.20 \times 10^{-4}$ & $4.68 \times 10^{-7}$ \\
TL-216 & 1.9080 & $1.00 \times 10^{-4}$ & $1.66 \times 10^{-6}$ & & 1.8285 & 0.0019 & $2.79 \times 10^{-6}$ \\
\hline
\end{tabular}

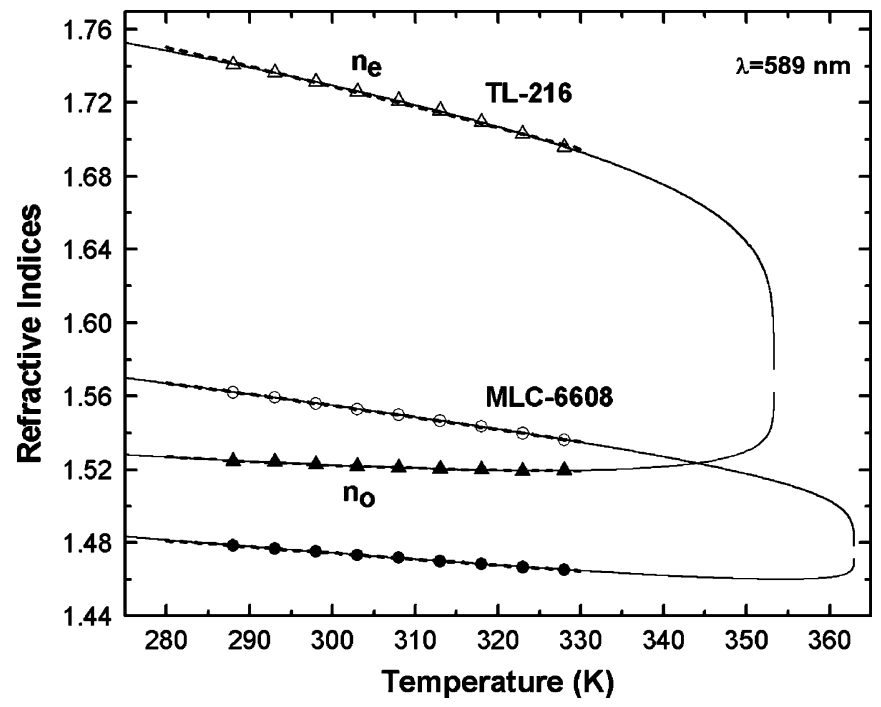

Fig. 6. Temperature-dependent refractive indices of MLC-6608 and TL-216 at $\lambda=589 \mathrm{~nm}$. The open and filled circles and triangles represent experimental data of $n_{e}$ and $n_{o}$ for MLC-6608 and TL-216, respectively. Solid and dashed lines are fittings using the four-parameter [see (14)] and the three-parameter parabolic [see (15)] models. The fitting parameters for the two models are listed in Tables VIII and IX, respectively.

\section{CONCLUSION}

We have derived and compared the three- and two-coefficient Cauchy models for describing the wavelength effect on the refractive indices of LCs based on the three-band model. If the LC birefringence is larger than $\sim 0.12$, the three-coefficient Cauchy model should be used. On the other hand, if the LC birefringence is smaller than $\sim 0.12$, the $\lambda^{-4}$ term can be ignored and the two-coefficient Cauchy model works equally well as the three-coefficient Cauchy model. Most of TFT LC mixtures developed for direct-view and projection displays have a relatively low birefringence. Thus, the two-coefficient Cauchy model is adequate. We have also derived and compared the four-parameter and three-parameter parabolic models for describing the temperature effect on the refractive indices of LCs based on the Vuks equation. The four-parameter model fits the experimental data very well in the whole nematic range. These four parameters can be obtained by two-stage fittings. The four-parameter model can be further simplified to the three-parameter parabolic model if the operating temperature is much lower than the clearing temperature. The three-parameter parabolic model

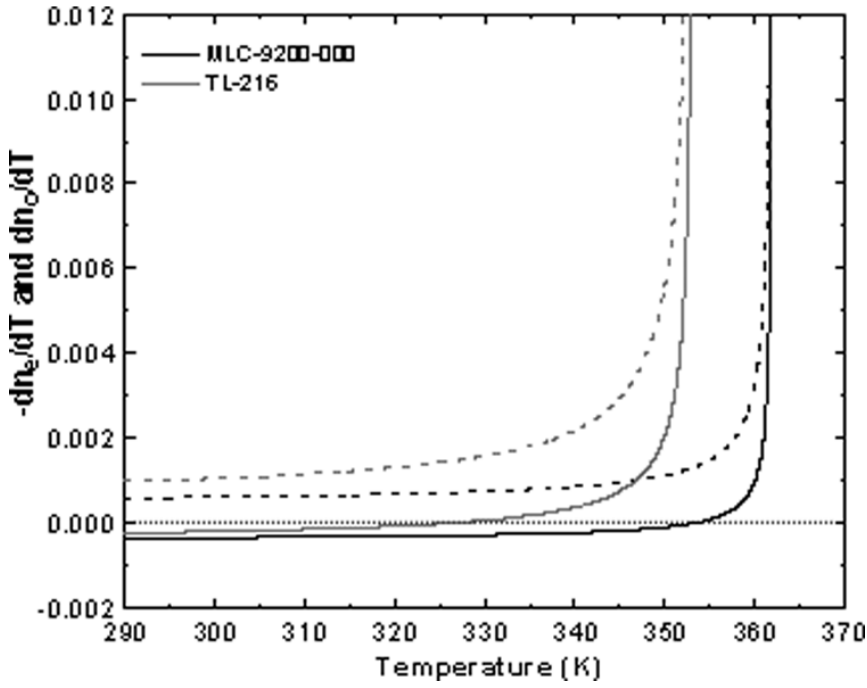

Fig. 7. Temperature gradient for $n_{e}$ and $n_{o}$ of MLC-9200-000 and TL-216 at $\lambda=546 \mathrm{~nm}$. Black and grey solid lines represent the calculated $d n_{o} / d T$ [see (16b)] curves for MLC-9200-000 and TL-216, respectively, while the dashed lines represent the calculated $-d n_{e} / d T$ [see (16a)] curves. The cross-over temperature for MLC-9200-000 and TL-216 are around 80.1 and 52.7 ${ }^{\circ} \mathrm{C}$, respectively.

works well for the four selected LC mixtures. Finally, the temperature gradients of $\mathrm{LC}$ refractive indices are presented. The $d n_{e} / d T$ remains negative throughout the nematic range. On the other hand, $d n_{o} / d T$ is negative when the temperature is below the crossover temperature $\left(T_{o}\right)$ but becomes positive when the temperature is beyond $T_{o}$. This indicates that $n_{e}$, the extraordinary refractive index, decreases monotonously as temperature increases throughout the nematic range whereas $n_{o}$, the ordinary refractive index, could decrease or increase with temperature depending on where the cross-over temperature is. Both $-d n_{e} / d T$ and $d n_{o} / d T$ change dramatically as the temperature approaches the clearing temperature.

\section{REFERENCES}

[1] E. H. Stupp and M. S. Brennesholtz, Projection Displays. New York: Wiley, 1998

[2] S. T. Wu and D. K. Yang, Reflective Liquid Crystal Displays. New York, NJ: Wiley, 2001.

[3] M. F. Vuks, "Determination of the optical anisotropy of aromatic molecules from the double refraction of crystals," Opt. Spektrosk., vol. 20, p. 644, 1966.

[4] W. H. de Jeu, Physical Properties of Liquid Crystalline Materials. New York: Gordon and Breach, 1980, ch. 4. 
[5] S. T. Wu, "Birefringence dispersions of liquid crystals," Phys. Rev. A, vol. 33, p. 1270, 1986.

[6] _ - "A semiempirical model for liquid-crystal refractive index dispersions," J. Appl. Phys., vol. 69, p. 2080, 1991.

[7] S. T. Wu, C. S. Wu, M. Warenghem, and M. Ismaili, "Refractive index dispersions of liquid crystals," Opt. Eng., vol. 32, p. 1775, 1993.

[8] E. M. Averyanov, "Dispersion of refractive indices in nematic liquid crystals," J. Opt. Technol., vol. 64, no. 5, p. 417, 1997.

[9] M. Born and E. Wolf, Principle of Optics, 6th ed. New York: Pergamon, 1980, ch. 2.

[10] H. Mada and S. Kobayashi, "Wavelength and voltage dependences of refractive indices of nematic liquid crystals," Mol. Cryst. Liq. Cryst., vol. 33, p. 47, 1976.

[11] L. Pohl and U. Finkenzeller, Liquid Crystals: Applications and Uses, B. Bahadur, Ed, Singapore: World Scientific, 1990, vol. 1, ch. 4.

[12] J. Li and S. T. Wu, "Extended Cauchy equations for the refractive indices of liquid crystals," J. Appl. Phys., vol. 95, p. 896, 2004.

[13] - "Two-coefficient Cauchy model for low birefringence liquid crystals," J. Appl. Phys., vol. 96, p. 170, 2004.

[14] J. Li, S. Gauza, and S. T. Wu, "Temperature effect on liquid crystal refractive indices," J. Appl. Phys., vol. 96, p. 19, 2004.

[15] V. Reiffenrath and M. Bremer, "First nematic calamitic liquid crystals with negative birefringence," Merck Liquid Crystal Newsletter, no. 15, p. 21,2000

[16] S. T. Wu and C. S. Wu, "Mixed-mode twisted nematic liquid crystal cell for reflective display," Appl. Phys. Lett., vol. 68, p. 1455, 1996.

[17] S. T. Wu, "Absorption measurements of liquid crystals in the ultraviolet, visible, and infrared," J. Appl. Phys., vol. 84, no. 8, p. 4462, 1998.

[18] I. C. Khoo and S. T. Wu, Optics and Nonlinear Optics of Liquid Crystals, Singapore: World Scientific, 1993.

[19] T. T. Alkeskjold, A. Bjarklev, D. S. Hermann, and J. Broeng, "Optical devices based on liquid crystal photonic bandgap fibers," Opt. Express, vol. 11 , p. $2589,2003$.

[20] T. T. Alkeskjold, J. Lægsgaard, A. Bjarklev, D. S. Hermann, D. S. Anawati, J. Li, and S. T. Wu, "All-optical modulation in dye-doped nematic liquid crystal photonic bandgap fibers," Opt. Express, vol. 12, p. 5857, 2004.

[21] M. Warenghem, J. F. Henninot, and G. Abbate, "Non linearly induced self waveguiding structure in dye doped nematic liquid crystals confined in capillaries," Opt. Express, vol. 2, p. 483, 1998.

[22] M. Warenghem, J. F. Henninot, F. Derrin, and G. Abbate, "Thermal and orientational spatial optical solitons in dye-doped liquid crystals," Mol. Cryst. Liq. Cryst., vol. 373, p. 213, 2002

[23] J. D. Jackson, Classical Electrodynamics, 2nd ed. New York: Wiley, 1962 , ch. 4

[24] J. Li and S. T. Wu, "Self-consistency of Vuks equations for liquid-crystal refractive indices," J. Appl. Phys., vol. 96, p. 6253, 2004

[25] J. Li, S. Gauza, and S. T. Wu, "High temperature-gradient refractive index liquid crystals," Opt. Express, vol. 12, p. 2002, 2004.

[26] I. Haller, "Thermodynamic and static properties of liquid crystals," Prog. Solid State Chem., vol. 10, p. 103, 1975.

[27] P. Janssen, J. A. Shimizu, J. Dean, and R. Albu, "Design aspects of a scrolling color LCoS display," Displays, vol. 23, p. 99, 2002.

[28] C. H. Wen, S. Gauza, and S. T. Wu, "Ultraviolet stability of liquid crystal alignment layers and mixtures," in Proc. SPIE, vol. 5740, 2005, pp. $67-75$.

[29] J. Li, S. T. Wu, S. Brugioni, S. Faetti, and R. Meucci, "Infrared refractive indices of liquid crystals," J. Appl. Phys., vol. 97, 2005.

[30] L. M. Blinov, Electro-Optical and Magneto-Optical Properties of Liquid Crystals. New York: Wiley, 1983.

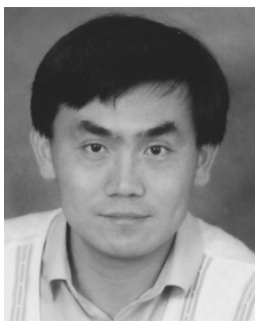

Jun Li (S'05) received the B.S. degree in mechanical engineering and the M.S. degree in automation from Tsinghua University, Beijing, China, in 1997 and 2001, respectively. He is currently working toward the Ph.D. degree from the College of Optics and Photonics/CREOL/FPCE, University of Central Florida, Orlando.

His current research is to develop structure-property correlation of liquid crystals (LCs), novel LC materials with high $\mathrm{dn}_{\mathrm{o}} / \mathrm{dT}$ at room temperature for highly thermal tunable LC photonic crystal bandgap fibers, and measurements of LC refractive indices in the visible and infrared spectral regions. His future work will focus on the polymer dispersed liquid crystal (PDLC), microdisplay technology, and novel LCs for display and photonic applications.

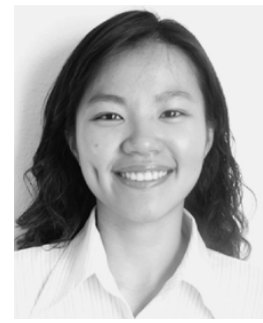

Chien-Hui Wen received the B.S degree in applied chemistry from National Chiao Tung University, Taiwan, in 2001, and M.S. degree in optics from University of Central Florida, Orlando, in 2003. She is currently working toward the Ph.D. degree from the College of Optics and Photonics: CREOL and FPCE, University of Central Florida.

Her current research activities focus on developing high performance negative dielectric anisotropy liquid crystals (LCs) and their alignment methods, and dual-frequency LC materials.

In 2005, Ms. Wen received the Newport Research Excellence Award.

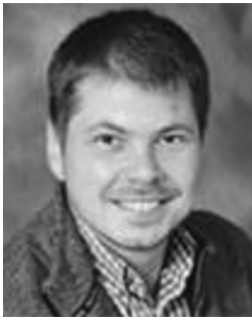

Sebastian Gauza received the Ph.D. in chemistry from the Military University of Technology in Warsaw, Poland, in 2001.

$\mathrm{He}$ is currently a research scientist at College of Optics and Photonics: CREOL and FPCE, University of Central Florida, Orlando. His current research is to develop novel high birefringence nematic liquid crystals, both single compounds and mixtures, for photonic applications. His future work will focus on novel nematic compositions for laser beam steering systems.

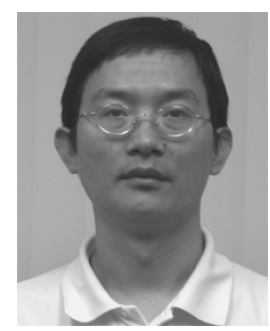

Ruibo Lu received the M.S degree in applied physics from Department of Physics, East China University of Science and Technology, Nanjing, China, in 1995, and the Ph.D. degree in optics from the Department of Physics, Fudan University, Shanghai, China, in 1998. His Ph.D. research work focused on liquid crystal (LC) alignment and ferroelectric LC devices for display and advanced optical applications.

$\mathrm{He}$ was a faculty in Department of Physics, and later in Department of Optical Science and Engineering, Fudan University, Shanghai, China, from 1998 to 2001. He was an optical engineer in Lightwaves2020 Inc., Milpitas, CA, from 2001 to 2002. Since 2002, he has been with the College of Optics and Photonics, University of Central Florida, as a research scientist. His research interests include liquid crystal (LC) display technology, wide viewing angle for LC TVs, LC components for optical communications, and optical imaging using LC medium.

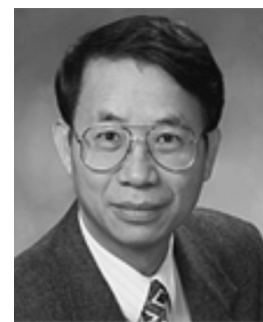

Shin-Tson Wu (M'98-SM'99-F'04) received the B.S. degree in physics from National Taiwan University, in 1975 and the Ph.D. degree from the University of Southern California, Los Angeles, in 1981.

$\mathrm{He}$ is a PREP professor at College of Optics and Photonics, University of Central Florida, (UCF), Orlando. His studies at UCF concentrate in foveated imaging, bio-photonics, optical communications, liquid crystal (LC) displays, and LC materials. Prior to joining UCF in 2001, he worked at Hughes Research Laboratories, Malibu, CA, for 18 years. He has coauthored two books: Reflective Liquid Crystal Displays (New York: Wiley, 2001) and Optics and Nonlinear Optics of Liquid Crystals (Singapore: World Scientific, 1993), four book chapters, and over 240 journal papers. He holds 23 U.S. patents.

Dr. Wu is a Fellow of the Society for Information Display (SID) and the Optical Society of America (OSA). 